\title{
Antidepressant-like Effects Induced by Chronic Blockade of the Purinergic 2X7 Receptor through Inhibition of Non-like Receptor Protein 1 Inflammasome in Chronic Unpredictable Mild Stress Model of Depression in Rats
}

\author{
Feyza Aricioglu' ${ }^{1}$, Ceren Sahin Ozkartal ${ }^{1}$, Tugce Bastaskin ${ }^{1}$, Erdem Tüzün${ }^{2}$, Cansu Kandemir ${ }^{3}$, Serap Sirvanci ${ }^{3}$, \\ Cem Ismail Kucukali ${ }^{2}$, Tijen Utkan ${ }^{4}$ \\ ${ }^{1}$ Department of Pharmacology and Psychopharmacology Research Unit, Marmara University School of Pharmacy, ${ }^{2}$ Department of Neuroscience, \\ Aziz Sancar Institute of Experimental Medical Research, Istanbul University, ${ }^{3}$ Department of Histology and Embryology, Marmara University \\ School of Medicine, Istanbul, ${ }^{4}$ Department of Pharmacology, Kocaeli University School of Medicine, Kocaeli, Turkey
}

\begin{abstract}
Objective: Purinergic $2 X 7$ receptor (P2X7R) activation is known to be involved in pathogenesis of depression. Our aims were to investigate P2X7R-activated inflammasome pathways in parallel with induction of depression and to test the antidepressant-like effects of the selective P2X7R antagonist Brilliant Blue G (BBG) in a rat model of chronic unpredictable mild stress (CUMS).

Methods: Male Wistar albino rats were divided into control, CUMS, CUMS + BBG25 $(25 \mathrm{mg} / \mathrm{kg} /$ day $)$ and CUMS+BBG50 $(50 \mathrm{mg} / \mathrm{kg} /$ day) groups ( $\mathrm{n}=10$ for each group). Various stressors were applied to rats for 6 weeks to establish the CUMS model and daily BBG treatment was started at the end of 3rd week. Sucrose preference test and forced swim test (FST) were performed to assess antidepressant-like effects. Brain samples were obtained for real-time polymerase chain reaction and immunohistochemistry analysis.

Results: In FST, duration of immobility was reduced in the CUMS+BBG50 group. Also, BBG treatment significantly enhanced sucrose preference. While NLRP3 gene expression levels were unchanged in rats exposed to the CUMS protocol, expression levels of other inflammasome pathway factors NLRP1, caspase-1, ASC, NF-KB, IL-1 $\beta$, IL-6 and P2X7R were increased. BBG treatment reduced expression levels of these factors. Likewise, Iba- 1 and GFAP immunoreactivities were enhanced by the CUMS protocol and this action was reversed by BBG treatment.

Conclusion: Chronic administration of BBG in CUMS model results in antidepressant-like activity in a dose dependent manner. Molecular and histological results show that these effects might be at least partially related to the suppression of inflammasome-related neuroinflammatory responses and suggest involvement of NLRP1 in depression.
\end{abstract}

KEY WORDS: Depression; Coomassie Brilliant Blue; ATP receptor; Purinergic P2X7 receptors; Animal models.

\section{INTRODUCTION}

Major depressive disorder (MDD) represents a serious and highly prevalent public health concern with a huge impact on the life quality of affected individuals. ${ }^{1-3)}$ One

Received: June 7, 2018/ Revised: July 11, 2018

Accepted: July 23, 2018

Address for correspondence: Erdem Tüzün, MD

Department of Neuroscience, Aziz Sancar Institute of

Experimental Medical Research, Istanbul University, Topkapı

Mahallesi, Vakıf Gureba Caddesi, Fatih 34390, Istanbul, Turkey

Tel: +90-212-4142000, Fax: +90-216-3452952

E-mail: drerdem@yahoo.com

ORCID: https://orcid.org/0000-0002-4483-0394 out of three MDD patients do not act in adequate response to existing pharmacological treatments, which mainly enhance monoaminergic neurotransmission. ${ }^{4,5)}$ This clearly indicates that the monoamine hypothesis alone is not sufficient to explain the pathogenesis of depression. Therefore, there is a certain need for novel antidepressant strategies and improved understanding of the underlying neurobiological mechanisms of depression.

Numerous studies have highlighted the association between depression and inflammation. ${ }^{6-9)}$ Patients with MDD have been reported to have higher levels of serum proinflammatory cytokines and acute phase reactants. ${ }^{10)}$

(ㄷ) This is an Open-Access article distributed under the terms of the Creative Commons Attribution Non-Commercial License (http://creativecommons.org/licenses/by-nc/4.0) which permits unrestricted non-commercial use, distribution, and reproduction in any medium, provided the original work is properly cited. 
Moreover, increased inflammatory mediators may serve as predictive biomarkers for resistance to antidepressant treatment. ${ }^{11)}$ Hence, treatment-resistant MDD patients with higher inflammatory mediators can actually benefit from anti-inflammatory medications. ${ }^{12,13)}$ Likewise, in experimental studies, administration of proinflammatory cytokines produces sickness behavior that resembles depression symptomatology in many aspects and anti-inflammatory approaches ameliorate depressive-like behaviors in animal depression models. ${ }^{14-17)}$ These findings have prompted researchers to better understand the mechanisms that trigger cytokine-associated inflammatory responses.

Most recently, inflammasome forming NOD-like receptor proteins (NLRP), a member of the pattern recognition receptors of the innate immune system, have been the subject of central nervous system (CNS) pathologies including depression for its particular role in initiation of interleukin 1 beta (IL-1 $\beta$ ) and IL-18 mediated inflammatory responses. ${ }^{18-20)}$ Among others, NLRP1 and NLRP3 are the best characterized members of NLRP family both of which expressed in the CNS. NLRP1 is mainly presented in neurons whereas NLRP3 is mostly located in microglia. ${ }^{21,22)}$ NLRP1 and NLRP3 inflammasomes are consist of apoptosis speck-like protein (ASC) and caspase-1 with the exception that NLRP1 inflammasome can also contain caspase-11. ${ }^{23)}$ NLRP1 and NLRP3 are normally inactive unless they encounter danger/pathogen associated stimulus. ${ }^{24)}$ Once activated, they form a multi-protein complex with ASC and pro-caspase-1, termed as the inflammasome, which relieves caspase- 1 , thereby leading to production and release of active forms of IL-1 $\beta$ and IL-18. ${ }^{22,25-27)}$

NLRP3 inflammasome activation and subsequent cytokine responses have been shown to be increased in animal depression models and MDD patients leading to the idea of NLRP3 inflammasome inhibition as a treatment model. ${ }^{16,17,28-30)}$ On the other side, the activation of NLRP1 inflammasome, which has been mainly implicated in neuronal injury pathologies including Alzheimer's disease or traumatic brain injury, has not been addressed in depression before. ${ }^{22,31)}$

The activation of NLRP depends upon adenosine triphosphate (ATP)-gated purinergic $2 \mathrm{X} 7$ receptor (P2X7R) signaling. ${ }^{20)}$ In fact, psychological stress can directly increase extracellular brain ATP levels which in turn acti- vates $P 2 X 7 R$ and results in inflammasome formation. ${ }^{26)}$ Thus, pharmacological blockade of P2X7R might be a therapeutic target for depression.

There is evidence that $\mathrm{P} 2 \mathrm{X} 7 \mathrm{R}$ gene polymorphisms may be associated with the development of depression. ${ }^{32-35)}$ The genetic deletion of the P2X7R or treatment with selective/non-selective $\mathrm{P} 2 \mathrm{XR} / \mathrm{P} 2 \mathrm{R}$ antagonists produce antidepressant-like effects in mice. ${ }^{36,37)}$ Finally, the acute administration of Brilliant Blue G (BBG) dye, a highly selective antagonist of $\mathrm{P} 2 \mathrm{X} 7$ receptors, ${ }^{38)}$ was shown to ameliorate bacterial lipopolysaccharide (LPS)-induced depression-like behaviors in mice. ${ }^{39)}$ However the antidepressant-like effects of antagonist substances used in these studies were mainly assessed by acute screening tests. Therefore, although these findings together support the involvement of purinergic signaling in depression, the antidepressant potential of chronic pharmacological blockade of P2X7R in well-validated chronic stress models of depression is still unknown.

Chronic unpredictable mild stress (CUMS) is considered to be one of the most extensively validated animal models of depression that meets good face, construct and predictive validity criteria. ${ }^{40,41)}$ As an environmental stress induced depression model, CUMS paradigm mimics many aspects of depression and it is especially well known for producing anhedonia-like behavior, which can be easily assessed by sucrose preference or sucrose consumption tests. ${ }^{42)}$ Furthermore, CUMS-induced depressive like behaviors only respond to chronic but not acute antidepressant treatments, which makes it one of the more realistic depression models. ${ }^{43-45)}$ Besides, the association between neuroinflammation, immune mechanisms and depression has been well studied in CUMS model. In this respect, CUMS-induced inflammation and inflammasome activation have been widely reported. $^{17-19,46,47)}$

Therefore, the present study was performed to examine the possible antidepressant-effects of chronic administration of BBG, a highly selective P2X7R antagonist, in conjunction with the investigation of NLRP inflammasome activation and neuroinflammatory responses.

\section{METHODS}

\section{Animals and Housing}

Adult male Wistar albino rats (8-10 weeks old) were ob- 
tained from Kocaeli University Experimental Medical Research and Application Center (DETAB, Kocaeli, Turkey) and were housed in groups of four per cage under standard laboratory conditions $\left(22 \pm 2^{\circ} \mathrm{C}\right.$ room temperature; 12-hour light/dark cycle with lights on at 7:00 AM and relative humidity of 55-50\%). Tap water and food pellets were provided ad libitum throughout the experiment. All experiments documented in this study were conducted in accordance with the Regulation of Animal Research Ethics Committee in Turkey (July 6, 2006, Number 26220). The Animal Research Ethics Committee of Marmara and Kocaeli University granted ethical approval. Before the experiments rats were allowed to habituate to the laboratory environment and the experimenters for 2 weeks.

\section{Drugs and Treatments}

BBG was purchased from Sigma Chemical Co. (St. Louis, MO, USA) and fresh BBG was dissolved in saline and given in a volume of $0.1 \mathrm{ml}$ per $100 \mathrm{~g}$ body weight of the rats. Fresh drug solutions were prepared on each day of behavioral testing. BBG was administered intraperitoneally (i.p.) in 25 and $50 \mathrm{mg} / \mathrm{kg}$ doses in every 24 hours during the last 3 weeks of CUMS. BBG doses were chosen based on previous studies demonstrating antidepressant-like effect of BBG in LPS-induced sickness model. ${ }^{39,48)}$ Control and CUMS groups received i.p. physiological saline for the last 3 weeks.

\section{Chronic Unpredictable Mild Stress Procedure and Experimental Design}

Rats were divided into 4 experimental groups ( $n=10$ / each group) including the control (non-stressed naïve healthy rats), CUMS, CUMS+BBG25 (BBG, $25 \mathrm{mg} / \mathrm{kg}$ ) and CUMS + BBG50 (BBG, 50 mg/kg) groups. CUMS procedure was applied for a total duration of 6 weeks and chronic treatment was started at the end of the 3rd week and continued throughout the CUMS on a daily basis. CUMS was applied as described previously. ${ }^{41,42)}$ Briefly, the CUMS groups w/wo treatments were subjected to nine different types of stressors, as listed: cage tilting for 24 hours, wet bedding for 24 hours, swimming in $4^{\circ} \mathrm{C}$ cold water for 5 minutes, swimming in $45^{\circ} \mathrm{C}$ hot water for $5 \mathrm{mi}$ nutes, pairing with another stressed animal for 48 hours, level shaking for 10 minutes, nip tail for 1 minute, and inversion of the light/dark cycle for 24 hours. These nine stressors were randomly applied for 6 weeks, and each stressor was applied 5 to 6 times during this period. Rats received only one of these stressors per day, and the same stressor was not applied on two consecutive days to prevent animals from predicting the occurrence of stimulation. The stress procedure did not involve any food or water deprivation. The control animals were kept in a separate experiment room and received no stress apart from saline injections and daily care. Body weights of rats were measured before and at the end of the 6-week treatment.

\section{Sucrose Preference Test}

Anhedonia-like behaviors were assessed by the sucrose preference test (SPT). In brief, each rat was placed in a test cage identical to home cage and was pre-exposed to the sucrose consumption test for 5 days for the adaptation period. The SPT was carried out on the sixth day of testing. Rats were housed individually and were exposed to two bottles, one containing $100 \mathrm{ml}$ of $20 \%$ sucrose and the other containing $100 \mathrm{ml}$ tap water, for a period of 1 hour after 23 hours of food and water deprivation. Water and sucrose intake and preference (\%) for sucrose (sucrose intake $[\mathrm{g}] /$ total fluid intake $[\mathrm{g}] \times 100)$ were calculated.

\section{Forced Swimming Test}

The forced swimming test (FST) apparatus was a cylinder (height, $47 \mathrm{~cm}$; inside diameter, $38 \mathrm{~cm}$ ) containing 38 $\mathrm{cm}$ of tap water maintained at $22 \pm 1^{\circ} \mathrm{C}$. The procedure was designed as previously described. ${ }^{49)}$ The experimental session consisted of two trials; conditioning and the test. During the conditioning trial, rats were gently placed into the cylinder and left in the water for 15 minutes. After the conditioning trial, rats were dried and placed into a warm cage with paper towels for 10 to 15 minutes before being returned to their home cages. The test trial was carried out 24 hours after the conditioning trial. Rats were placed again into the cylinder and left in the water for a 5-minute test session. After the test session, rats were removed from the cylinder, and dried with a towel before being returned to their home cages. Tests were videotaped. The immobility time, which was defined as the lack of motion of the whole body except for the small movements necessary to keep the animal's head above the water, was recorded. An observer blind to the treatment conditions recorded the time spent immobile in the test session. 


\section{Real-time Polymerase Chain Reaction (PCR) Analysis}

Frozen prefrontal cortex tissues were homogenized and total RNA was extracted using a commercial RNAzol RT isolation kit (Molecular Research Center, Inc., Cincinnati, $\mathrm{OH}$, USA). RNA concentrations were spectrophotometrically determined. The purification and the concentration of $1 \mu \mathrm{l}$ RNA samples were assessed by 260/280 and 260/230 ratios. Complementary DNA (cDNA) synthesis from RNA samples were performed with commercial cDNA synthesis kit (Jena Bioscience, Jena, Germany; Cat no. PCR511). Two microliters of the cDNA sample was used along with qPCR GreenMaster kit (Jena Bioscience; Cat no: PCR306) for real-time reverse transcription PCR. The primers used in the study were obtained from DNA Technology (Moscow, Russia) and were shown in Table 1 . Beta-actin ( $\beta$-actin) was used as an internal control (housekeeping) gene. The cycle of threshold (CT) of investigated primers was determined and normalized to housekeeping gene, $\beta$-actin. Relative quantitation was calculated with $2^{\wedge}-(\mathrm{ddCT})$ method and data are presented as relative changes to control group.

\section{Perfusion Fixation and Tissue Preparation}

For perfusion fixation, $4 \%$ paraformaldehyde in $0.1 \mathrm{M}$ phosphate-buffered saline ( $\mathrm{pH}$ 7.4) was used. After decapitation, brain tissues were obtained and incubated in the same fixative overnight at $4^{\circ} \mathrm{C}$. Tissues were dehydrated and cleared in a tissue processor (Leica TP1020l; Leica Biosystems, Wetzlar, Germany). After incubating in liquid paraffin at $60^{\circ} \mathrm{C}$ overnight, the tissues were embedded in paraffin in a tissue embedder.

\section{Immunohistochemistry}

Due to the extensive panel of inflammation mediators, the entire prefrontal cortex was used for real time PCR analysis. Therefore, hippocampus, another brain region afflicted from depression was used for immunohistochemistry studies. Five-micron-thick paraffin sections were obtained. After deparaffinization, sections were incubated in descending series of ethanol and treated with $3 \% \mathrm{H}_{2} \mathrm{O}_{2}$. For antigen retrieval, sodium citrate buffer was used. Sections were incubated with blocking solution, and then with anti-ionized calcium-binding adapter molecule (Iba-1) antibody (ab108539; Abcam, Cambridge, UK) and anti-glial fibrillary acidic protein (GFAP) antibodies (MAB3402; Millipore, Burlington, MA, USA) at $37^{\circ} \mathrm{C}$ for 1 hour. After applying biotinylated secondary antibody and horseradish peroxidase streptavidin (Histostain Plus Broad Spectrum 859043 and 859043; Invitrogen, Carlsbad, CA, USA), reaction was observed using 3,3'-diaminobenzidine (DAB Plus Substrate Kit 002020; Invitrogen). Mayer's hematoxylin was used for counterstaining. Sections were mounted and coverslipped with Entellan. Five areas from the dorsal hippocampus were evaluated for counting Iba-1 or GFAP immunoreactive cells under a microscope by using $40 \times$ objective.

\section{Statistical Analysis}

GraphPad Prism Program (GraphPad Software Inc., La Jolla, CA, USA) was used for statistical calculations. Results were expressed as mean \pm standard error of mean. One-way analysis of variance (ANOVA) was used for statistical comparisons between groups. The Tukey's honestly significant difference test was used for post hoc analysis. Pearson test was used to assess potential correlations among mRNA expression levels vs. FST and SPT test results. A $p$ value less than 0.05 was considered as a value of significance.

Table 1. Primers used in real-time RT-PCR analysis

\begin{tabular}{lll}
\hline Gene & \multicolumn{1}{c}{ Forward primer } & \multicolumn{1}{c}{ Reverse primer } \\
\hline$N L R P 3$ & CCATGAGCTCCCTTAAGCTG & TTGCACAGGATCTTGCAGAC \\
$N L R P 1$ & GTTGCAAGTCCCTTCAGCTC & CATCTCTGTTTCCGAGCACA \\
Caspase- 1 & GCTTGAAAGACAAGCCCAAG & CCTTTCAGTGGTTGGCATCT \\
ASC & GCAATGTGCTGACTGAAGGA & TGTTCCAGGTCTGTCACCAA \\
$N F-\kappa B$ & GGCTGACCTGAGTCTTCTGG & GATAAGGAGTGCTGCCTTGC \\
$I L-1 \beta$ & AGGCTTCCTTGTGCAAGTGT & TGAGTGACACTGCCTTCCTG \\
$L L-6$ & CCGGAGAGGAGACTTCACAG & ACAGTGCATCATCGCTGTTC \\
$P 2 X 7 R$ & GTGGAGACGGTGAAGGTGTT & AACGACACCTTTGGTCTTG \\
$\beta$-aCtin & GCCCCCGGTTTCTATAAATTG & GTCGAACAGGAGGAGCAGAGA \\
\hline
\end{tabular}

RT-PCR, reverse transcription-polymerase chain reaction. 


\section{RESULTS}

\section{The Effect of CUMS and BBG Treatments on Clinical Parameters}

There were no significant differences between the baseline body weights of study groups. By contrast, at the end of the study, the body weights of rats in the CUMS group was significantly reduced, whereas those of control, CUMS+BBG25 and BBG50 groups were increased ( $p=$ 0.037 by ANOVA; Table 2).

As shown in Figure 1, rats exposed to 6-week CUMS procedure developed anhedonia-like behavior with marked decreases in sucrose preference compared to non- stressed control group $(p<0.01)$ in SPT. Chronic administration of BBG (25 and $50 \mathrm{mg} / \mathrm{kg}$ ) during the last 3 weeks of CUMS procedure significantly increased sucrose preference compared to CUMS group $(p<0.01)$. Our results show that BBG treatment reversed CUMS-induced

Table 2. Body weights of rats at baseline and at the end of the study period

\begin{tabular}{lll}
\hline \multicolumn{1}{c}{ Group } & Baseline $(\mathrm{g})$ & 6th week $(\mathrm{g})$ \\
\hline Control & $276.5 \pm 11.9$ & $290.3 \pm 12.5$ \\
CUMS & $292.6 \pm 8.7$ & $271.5 \pm 8.5$ \\
CUMS+BBG25 & $210.8 \pm 4.7$ & $281.5 \pm 4.9$ \\
CUMS+BBG50 & $221.0 \pm 41$ & $284.2 \pm 5.4$ \\
\hline
\end{tabular}

Values are presented as mean \pm standard error.

CUMS, chronic unpredictable mild stress; BBG25, Brilliant Blure G $25 \mathrm{mg} / \mathrm{kg} /$ day; BBG50, BBG $50 \mathrm{mg} / \mathrm{kg} /$ day.

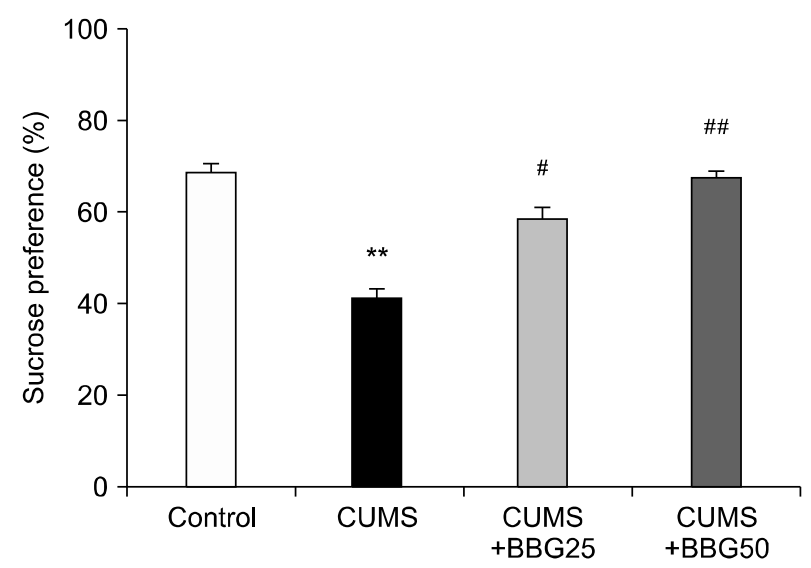

Fig. 1. The effect of chronic unpredictable mild stress (CUMS) procedure and chronic Brilliant Blue G (BBG) treatment on anhedonia-like behaviors of rats in sucrose preference test. BBG25, BBG 25 mg/kg/day; BBG50, BBG 50 mg/kg/day. Data are expressed as mean \pm stadard error of mean ( $n=10 /$ each group). ${ }^{* *} p<0.01$ vs. control group; ${ }^{\#} p<0.05$ and ${ }^{\# \#} p<0.01$ vs. CUMS group. anhedonia-like behaviors in rats (Fig. 1).

In the FST, the time of immobility was significantly elevated in CUMS group compared to control group $(p<$ 0.01). However, rats in the CUMS + BBG50 group ( $p<$ 0.01 ) but not CUMS+BBG25 group had significantly shorter duration of immobility showing that higher dose of BBG was effective in improving CUMS-induced despair-like behaviors of rats (Fig. 2).

\section{The Effect of CUMS and BBG Treatments on Gene Expression Levels of NLRP Cascade and Relevant Neuroinflammatory Components}

We measured an array of certain neuroinflammatory mediators implicated in NLRP inflammasome activation and cytokine responses in prefrontal cortex of rats. The first cluster of parameters was composed of inflammasome cascade including two major inflammasome forming NLRP (NLRP1 and NLRP3) and related components; ASC, and caspase- 1 , in addition to nuclear factor kappa B $(\mathrm{NF}-\kappa \mathrm{B})$ and proinflammatory cytokines; IL-1 $\beta$ and IL-6 and P2X7R expression profile. Secondly we investigated CD-11b and Iba-1 as markers for microglial activation and astrocyte GFAP expression.

Real-time PCR analysis showed that P2X7R mRNA levels were markedly increased in CUMS exposed rats relative to control group $(\mathrm{F}=10.90, p<0.05)$. Besides, chronic blockage of $P 2 X 7 R$ by BBG (25 and $50 \mathrm{mg} / \mathrm{kg}$ ) downregulated $P 2 X 7 R$ gene expressions $(p<0.01)$ (Fig. $3 A)$. In

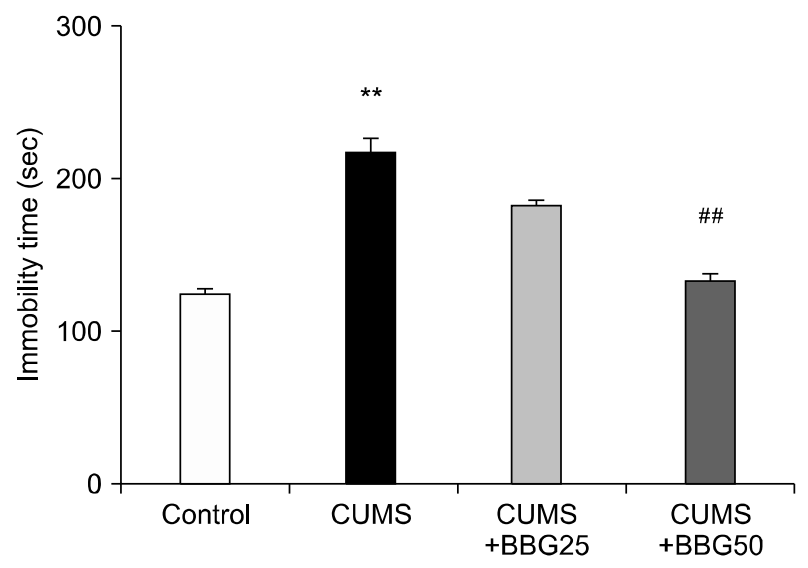

Fig. 2. The effect of chronic unpredictable mild stress (CUMS) procedure and chronic Brilliant Blue G (BBG) treatment on despair-like behaviors of rats in forced swim test.

BBG25, BBG 25 mg/kg/day; BBG50, BBG 50 mg/kg/day.

Data are expressed as mean \pm stadard error of mean ( $n=10 /$ each group). ${ }^{* *} p<0.01$ vs. control group; ${ }^{\# \#} p<0.01$ vs. CUMS group. 

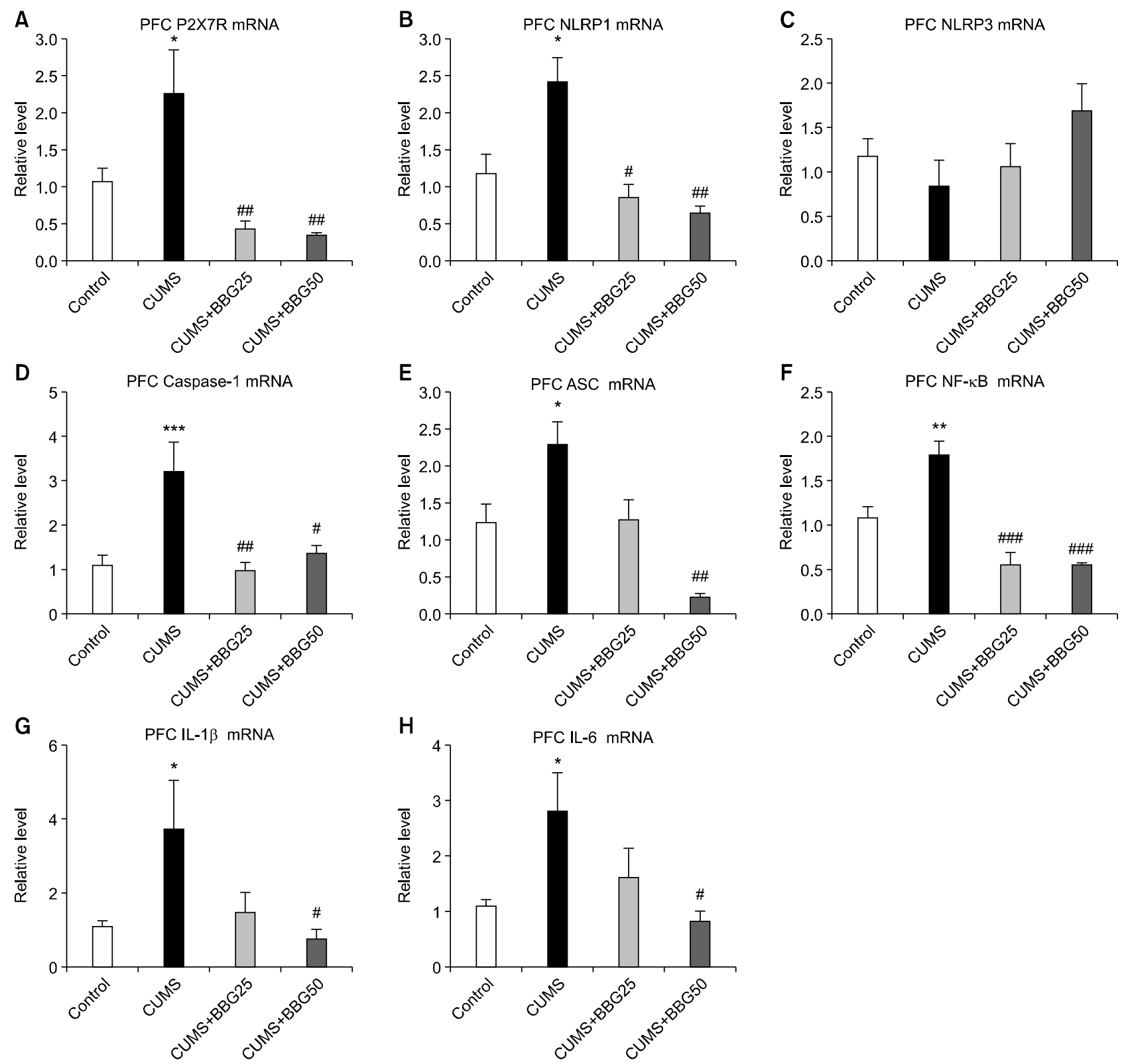

Fig. 3. The effect of chronic unpredictable mild stress (CUMS) procedure and chronic Brilliant Blue G (BBG) treatment on mRNA levels of NOD-like receptor protein (NLRP) inflammasome components and relevant neuroinflammatory mediators in prefrontal cortex (PFC) of rats. ASC, apoptosis speck like protein; IL, interleukin; NF-kB, nuclear factor kappa B; BBG25, BBG 25 mg/kg/day; BBG50, BBG 50 mg/kg/day. Data are expressed as mean \pm stadard error of mean ( $\mathrm{n}=5 /$ each group).

${ }^{*} p<0.05,{ }^{* *} p<0.01,{ }^{* * *} p<0.001$ vs. control group; ${ }^{\#} p<0.05,{ }^{\# \#} p<0.01,{ }^{\# \# \#} p<0.001$ vs CUMS group.

accordance with $P 2 X 7 R$ gene expressions, we found that 6-week CUMS procedure caused significant elevations in mRNA levels of inflammasome forming protein, NLRP1 $(\mathrm{F}=6.738, p<0.05)$ but not NLRP3 (Fig. 3B, 3C). CUMS + BBG25 $(p<0.05)$ and CUMS + BBG50 groups $(p<0.01)$ had significantly reduced $N \angle R P 1 \mathrm{mRNA}$ levels in the prefrontal cortex (Fig. 3B). Although NLRP3 levels were not affected by CUMS, CUMS+BBG50 group showed sig- nificantly higher NLRP3 levels compared to CUMS alone ( $\mathrm{F}=4.219, p<0.05)$ (Fig. 3C).

In addition to NLRP1 and NLRP3, we investigated two other molecular components, caspase- 1 and ASC, that together participate in inflammasome formation and therefore, playing a crucial role in initiating IL-1 $\beta$ mediated inflammatory responses. We found that relative mRNA levels of caspase-1 were significantly elevated in prefrontal 
cortex of CUMS-treated rats compared to controls $(\mathrm{F}=$ 8.756, $p<0.001)$. This effect was reversed in CUMS+ BBG25 $(p<0.01)$ and CUMS + BBG50 $(p<0.05)$ treatment groups (Fig. 3D). Likewise, relative mRNA levels of $A S C$, an adaptor protein for inflammasome formation, were elevated by CUMS procedure ( $\mathrm{F}=6.449, p<0.05)$. Treatment with $50 \mathrm{mg} / \mathrm{kg}(p<0.01)$ but not $25 \mathrm{mg} / \mathrm{kg}$ BBG attenuated CUMS-induced ASC mRNA levels in prefrontal cortex of rats (Fig. 3E).

We also investigated the possible changes in mRNA levels of $N F-\kappa B$, an important transcriptional factor in the inflammatory cascade, in response to CUMS and BBG treatment. It was found that CUMS-induced $N F-\kappa B$ levels $(\mathrm{F}=15.77, p<0.01)$ were alleviated in CUMS+BBG25

A

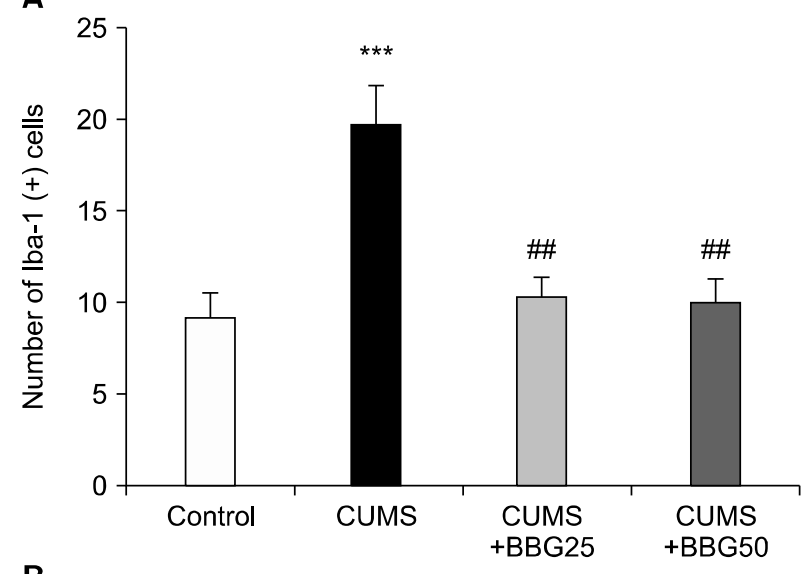

B

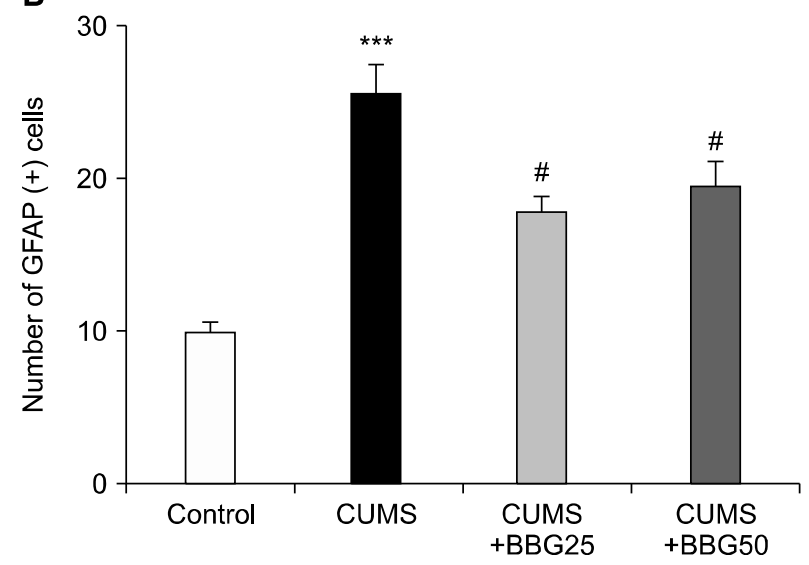

and CUMS + BBG50 groups $(p<0.001)$ (Fig. 3F). CUMS exposure for 6 weeks caused significant elevations in relative gene expressions of two major pro-inflammatory cytokines, $I L-1 \beta(\mathrm{F}=3.810, p<0.05)$ and $/ L-6(\mathrm{~F}=4.889, p<$ $0.05)$, that are known to be highly implicated in depression. When administered only at the highest dose, BBG significantly reduced the mRNA levels of $/ L-1 \beta(p<0.05)$ and $I L-6(p<0.05)$ (Fig. 3G, 3H). In the CUMS group, significant negative correlation was found among SPT values vs $/ L-6(p=0.017, \mathrm{R}=-0.991)$ and $/ L-1 \beta \mathrm{mRNA}(p=0.042$, $\mathrm{R}=-0.964)$ levels and a positive correlation was found among FST immobility time vs $N F-\kappa B$ mRNA levels $(p=0.004, \mathrm{R}=0.999)$. No significant correlation could be found in control and BBG treatment groups.
C
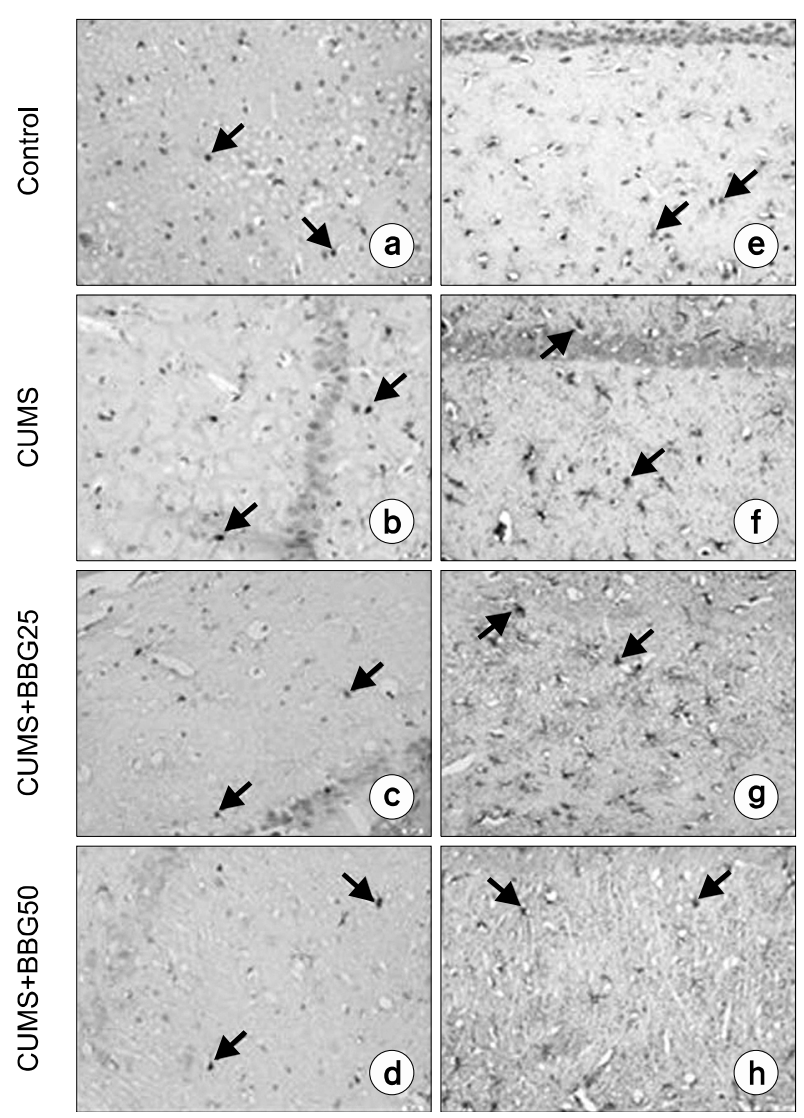

Fig. 4. The effect of chronic unpredictable mild stress (CUMS) procedure and chronic Brilliant Blue G (BBG) treatment on microglia and astroglial activation. (A) Number of Iba-1 (+) microglial cells in the dorsal hippocampus. (B) Number of GFAP $(+)$ astrocytes in the dorsal hippocampus. (C) Iba-1 (a-d) and GFAP (e-h) immunostaining: (a, e) control, (b, f) CUMS, (c, g) CUMS+BBG25, and (d, h) CUMS+BBG50. Arrows, Iba-1 (a-d) or GFAP (e-h) immunoreactive microglia (a-d) or astrocytes (e-h). Original magnification, $\times 400$.

Iba-1, ionized calcium-binding adapter molecule-1; GFAP, glial fibrillary acidic protein; PFC; prefrontal cortex; BBG25, BBG 25 mg/kg/day; BBG50, BBG $50 \mathrm{mg} / \mathrm{kg} /$ day.

Data are expressed as mean \pm stadard error of mean ( $\mathrm{n}=5 /$ each group).

${ }^{* * *} p<0.001$ vs. control group; ${ }^{\#} p<0.05,{ }^{\# \#} p<0.01$ vs. CUMS group. 


\section{The Effect of CUMS and BBG Treatments on Microglial and Astroglial Activation in Hippocampus}

Iba-1 immunoreactivity was assessed in hippocampal brain tissue of rats and it was found to be significantly increased in CUMS group compared to non-stressed control group $(p<0.001)$. Chronic treatment with BBG in both dosage attenuated CUMS-induced hippocampal Iba (+) immunoreactive cells ( $p<0.01$ ) (Fig. 4A, 4C). Besides, we showed that CUMS procedure caused significantly increased GFAP expression $(p<0.001)$, whereas treatment with both 25 and $50 \mathrm{mg} / \mathrm{kg}$ of BBG $(p<0.05)$ resulted in significant reduction in GFAP $(+)$ astrocyte expression induced by CUMS ( $p<0.05$ ) (Fig. 4B, 4C).

\section{DISCUSSION}

In the present study, the antidepressant effect of BBG, a selective antagonist of the $\mathrm{P} 2 \mathrm{X} 7 \mathrm{R}$, was investigated in CUMS model of depression. Our study has demonstrated that 6-week CUMS procedure induced depressive-like behaviors that were coupled with increased P2X7R signaling, NLRP inflammasome activation and glial activation. Moreover, in rats exposed to the CUMS model, higher $N F-\kappa B$ mRNA levels were associated with increased immobility time in FST and higher $I L-6$ and $I L-1 \beta$ mRNA levels were associated with reduced sucrose preference rates. These CUMS model-induced effects were annulled by BBG treatment. In brief, chronic administration of BBG induced an antidepressant-like effect at least partially via inhibition of NLRP1 inflammasome-mediated neuroinflammatory processes.

It is possible to generate certain aspects of depression symptomatology (such as anhedonia, despair and helplessness) by CUMS model of depression. ${ }^{40,42,50)}$ Anhedonia, one of the main symptoms of depressive disorder, is commonly validated by a decrease in sucrose preference. ${ }^{45)}$ In the present study, 6-week CUMS paradigm led to a decrease in sucrose preference in rats. Chronic treatment with both $25 \mathrm{mg} / \mathrm{kg}$ and $50 \mathrm{mg} / \mathrm{kg}$ BBG restored sucrose preference back to normal values. In a recent study, subacute administration of $50 \mathrm{mg} / \mathrm{kg}$ of BBG for 4 days was shown to reverse LPS-induced anhedonia in mice. ${ }^{48)}$ Thus we have obtained similar results in Wistar albino rats by chronic administration of BBG.

In addition to anhedonia, it is well reported that despair/helplessness-like behavior, which is another im- portant feature of depression is triggered by CUMS procedure. This aspect of depression can be assessed by FST. ${ }^{29,51)}$ CUMS-exposed rats remained motionless for a longer time than non-stressed healthy controls, thereby confirming induction of despair-like state. Administration of $50 \mathrm{mg} / \mathrm{kg}$ BBG reversed this CUMS-induced symptom. It has recently been shown in mice that single administration of BBG at $50 \mathrm{mg} / \mathrm{kg}$ attenuates LPS-induced immobility time in FST. ${ }^{39)}$ These results are also compatible with previous studies conducted with other $\mathrm{P} 2 \mathrm{R} / \mathrm{P} 2 \mathrm{XR}$ antagonists and P2X7R knockout mice. ${ }^{36,52)}$ Therefore, our findings further confirm the antidepressant effect of BBG. Moreover, we herein show for the first time that P2X7R antagonism does not only ameliorate acutely induced depression with a single dosing but also chronic environmental stress induced depression with a prolonged treatment regimen.

Studies using different depression models have shown a cortical P2X7R upregulation, which has been reversed by BBG treatment. ${ }^{53,54)}$ Moreover, BBG administered intravenously after traumatic brain injury has significantly reduced enhanced P2X7R and IL-1 $\beta$ expression in the rat cortex. ${ }^{55)}$ Similarly, in our study, exposure to CUMS induced an increase in P2X7R and inflammasome pathway expression levels, which was reversed by BBG treatment in parallel to amelioration in depressive symptoms. Thus our results further confirm the link between P2X7R-mediated inflammasome activation and depression.

NLRP3 inflammasome has lately been the focus of depression research. ${ }^{14)}$ Several studies have shown NLRP3 inflammasome activation in animal depression models and patients with MDD. ${ }^{51,53,56-59)}$ However, the possible involvement of NLRP1 inflammasome activation in depression has not been addressed before. Our 6-week CUMS procedure caused significant upregulation of NLRP1, ASC and caspase-1, indicating involvement of the NLRP1 inflammasome in depression.

The lack of alteration in NLRP3 mRNA expression in CUMS-exposed rats of our study might be due to the differences in CUMS protocols or utilized animal strains across different studies. Besides, post-transcriptional regulation of NLRP3 cannot be excluded and therefore protein expression analyses in specific brain regions are required for a better interpretation. It is of note that, the vast majority of the literature investigating the involvement of NLRP3 inflammasome in depression or stress has reported 
upregulation via determination of protein expression levels. ${ }^{26,28,29,60)}$ In fact, only a small number of studies has examined the gene expression levels of NLRP3. However, each of these studies has used different depression models. For instance, Zhang et al. ${ }^{61)}$ demonstrated increased NLRP3 mRNA levels in LPS-induced sickness model in mice. Our group and also others have reported subacute/chronic restraint stress to enhance NLRP3 mRNA levels in prefrontal cortex and hippocampus of rats. ${ }^{56,62)}$ Only in one study using the CUMS protocol, an increase in NLRP3 mRNA levels was shown in prefrontal cortex of rats. ${ }^{53)}$ However, the duration of CUMS procedure was rather long (12-week) and the stressors differed from our study. Overall, these results suggest that depression-induced inflammasome pathway activation is contingent upon duration and type of stressors used in depression models.

NF- $\kappa \mathrm{B}$ is a transcription factor producing proinflammatory cytokines such as IL-6, tumor necrosis factor-alpha, IL-1 $\beta$ and IL18. ${ }^{63,64)}$ In our study, CUMS-exposed rats showed higher mRNA levels of $N F-\kappa B, I L-1 \beta$ and IL- 6 , which were reduced by chronic treatment with BBG. Our results thus confirm the cytokine reducing action of BBG previously established in LPS-induced depression model. $^{39,54)}$

Another important effect of P2X7R antagonism is reduction of enhanced microglial and astrocytic activity as shown by reduced Iba- 1 and GFAP immunoreactivity. Increased glial activation has been shown in other chronic stress models of depression including CUMS. ${ }^{65,66)}$ BBG administration has been shown to attenuate enhanced glial activity in ischemic and traumatic brain and spinal cord injury models. ${ }^{67,68)}$ We herein, show in a well-established animal model that BBG also effectively inhibits depression-induced gliosis. Glial cells may interfere with neuronal functions by secreting not only cytokines but also other toxic mediators such as free radical producing enzymes and metalloproteinases, thereby ultimately causing cognitive dysfunction. ${ }^{69)}$ Therefore, our results emphasize preservation of the neuronal reserves of depression patients as another beneficial action of P2X7R antagonism.

In conclusion, chronic administration of a highly selective P2X7R antagonist, BBG, produces antidepressantlike effects in a well-validated chronic depression model. This action is at least partially mediated by suppression of enhanced NLRP1 inflammasome gene expression levels, proinflammatory cytokine responses and glial activation. Therefore, we suggest that $\mathrm{P} 2 \mathrm{X} 7 \mathrm{R}$ inhibition may provide a potential therapeutic target for inflammatory aspect of depression.

\section{- Acknowledgments}

This study was supported by Scientific Research Project Unit of Marmara University (Project no: SAG-C-YLP110915-0416 and SAG-E-120613-0233), Scientific Research Projects Coordination Unit of Istanbul University (Project no: 22790) and The Scientific and Technological Research Council of Turkey (TÜBİTAK) (Project no: 216S797).

\section{REFERENCES}

1. Kessler RC. The costs of depression. Psychiatr Clin North Am 2012;35:1-14.

2. Menken M, Munsat TL, Toole JF. The global burden of disease study: implications for neurology. Arch Neurol 2000;57:418420.

3. Murray CJ, Lopez AD. Alternative projections of mortality and disability by cause 1990-2020: global burden of disease study. Lancet 1997;349:1498-1504.

4. Culpepper L. Why do you need to move beyond first-line therapy for major depression? J Clin Psychiatry 2010;71 Supp/ 1:4-9.

5. Sahin C, Dursun S, Cetin M, Aricioglu F. The neuroinflammation perspective of depression: reuniting the outstanding mechanisms of the pathophysiology. Klin Psikofarmakol Bülteni Bull Clin Psychopharmacol 2016;26: 196-206.

6. Ferrari P, Parisi MM, Colombo R, Becker M, Fries G, Ascoli $\mathrm{BM}$, et al. Depression and mania induce pro-inflammatory activation of macrophages following application of serum from individuals with bipolar disorder. Clin Psychopharmacol Neurosci 2018;16:103-108.

7. Réus GZ, Fries GR, Stertz L, Badawy M, Passos IC, Barichello $\mathrm{T}$, et al. The role of inflammation and microglial activation in the pathophysiology of psychiatric disorders. Neuroscience 2015;300:141-154.

8. Barbosa IG, Vaz GN, Rocha NP, Machado-Vieira R, Ventura MRD, Huguet RB, et al. Plasma levels of tumor necrosis factor superfamily molecules are increased in bipolar disorder. Clin Psychopharmacol Neurosci 2017;15:269-275.

9. Miller $\mathrm{AH}$, Maletic $\mathrm{V}$, Raison $\mathrm{CL}$. Inflammation and its discontents: the role of cytokines in the pathophysiology of major depression. Biol Psychiatry 2009;65:732-741.

10. Miller $\mathrm{AH}$, Raison CL. The role of inflammation in depression: from evolutionary imperative to modern treatment target. Nat Rev Immunol 2016;16:22-34. 
11. Strawbridge R, Arnone D, Danese A, Papadopoulos A, Herane Vives A, Cleare AJ. Inflammation and clinical response to treatment in depression: a meta-analysis. Eur Neuropsychopharmacol 2015;25:1532-1543.

12. Dean OM, Maes M, Ashton M, Berk L, Kanchanatawan B, Sughondhabirom A, et al. Protocol and rationale-the efficacy of minocycline as an adjunctive treatment for major depressive disorder: a double blind, randomised, placebo controlled trial. Clin Psychopharmacol Neurosci 2014;12:180-188.

13. Raison CL, Rutherford RE, Woolwine BJ, Shuo C, Schettler P, Drake DF, et al. A randomized controlled trial of the tumor necrosis factor antagonist infliximab for treatment-resistant depression: the role of baseline inflammatory biomarkers. JAMA Psychiatry 2013;70:31-41.

14. Iwata M, Ota KT, Duman RS. The inflammasome: pathways linking psychological stress, depression, and systemic illnesses. Brain Behav Immun 2013;31:105-114.

15. Wei L, Kan LY, Zeng HY, Tang YY, Huang HL, Xie M, et al. $B D N F / T r k B$ pathway mediates the antidepressant-like role of H2S in CUMS-exposed rats by inhibition of hippocampal ER stress. Neuromolecular Med 2018;20:252-261.

16. Song MT, Ruan J, Zhang RY, Deng J, Ma ZQ, Ma SP. Astragaloside IV ameliorates neuroinflammation-induced depressive-like behaviors in mice via the PPAR y NF- KB/NLRP3 inflammasome axis. Acta Pharmacol Sin 2018;39:1559-1570.

17. Song Y, Sun R, Ji Z, Li X, Fu Q, Ma S. Perilla aldehyde attenuates CUMS-induced depressive-like behaviors via regulating TXNIP/TRX/NLRP3 pathway in rats. Life SCi 2018;206:117124.

18. Kaufmann FN, Costa AP, Ghisleni G, Diaz AP, Rodrigues ALS, Peluffo $\mathrm{H}$, et al. NLRP3 inflammasome-driven pathways in depression: clinical and preclinical findings. Brain Behav Immun 2017;64:367-383.

19. Haapakoski R, Ebmeier KP, Alenius H, Kivimäki M. Innate and adaptive immunity in the development of depression: an update on current knowledge and technological advances. Prog Neuropsychopharmacol Biol Psychiatry 2016;66:63-72.

20. Velasquez S, Rappaport J. Inflammasome activation in major depressive disorder: a pivotal linkage between psychological stress, purinergic signaling, and the kynurenine pathway. Biol Psychiatry 2016;80:4-5.

21. Kummer JA, Broekhuizen R, Everett H, Agostini L, Kuijk L, Martinon $\mathrm{F}$, et al. Inflammasome components NALP 1 and 3 show distinct but separate expression profiles in human tissues suggesting a site-specific role in the inflammatory response. J Histochem Cytochem 2007;55:443-452.

22. Kigerl KA, de Rivero Vaccari JP, Dietrich WD, Popovich PG, Keane RW. Pattern recognition receptors and central nervous system repair. Exp Neurol 2014;258:5-16.

23. de Rivero Vaccari JP, Dietrich WD, Keane RW. Activation and regulation of cellular inflammasomes: gaps in our knowledge for central nervous system injury. J Cereb Blood Flow Metab 2014;34:369-375.
24. Di Virgilio F. The therapeutic potential of modifying inflammasomes and NOD-like receptors. Pharmacol Rev 2013; 65:872-905.

25. Guarda G, So A. Regulation of inflammasome activity. Immunology 2010;130:329-336.

26. Iwata M, Ota KT, Li XY, Sakaue F, Li N, Dutheil S, et al. Psychological stress activates the inflammasome via release of adenosine triphosphate and stimulation of the purinergic type $2 X 7$ receptor. Biol Psychiatry 2016;80:12-22.

27. Fleshner M, Frank M, Maier SF. Danger signals and inflammasomes: stress-evoked sterile inflammation in mood disorders. Neuropsychopharmacology 2017;42:36-45.

28. Zhang Y, Liu L, Liu YZ, Shen XL, Wu TY, Zhang T, et al. NLRP3 inflammasome mediates chronic mild stress-induced depression in mice via neuroinflammation. Int J Neuropsychopharmacol 2015;18:pyv006.

29. Liu B, Xu C, Wu X, Liu F, Du Y, Sun J, et al. Icariin exerts an antidepressant effect in an unpredictable chronic mild stress model of depression in rats and is associated with the regulation of hippocampal neuroinflammation. Neuroscience 2015;294:193-205.

30. Alcocer-Gómez E, Ulecia-Morón C, Marín-Aguilar F, Rybkina T, Casas-Barquero N, Ruiz-Cabello J, et al. Stress-induced depressive behaviors require a functional NLRP3 inflammasome. Mol Neurobiol 2016;53:4874-4882.

31. Freeman LC, Ting JP. The pathogenic role of the inflammasome in neurodegenerative diseases. I Neurochem 2016;136 Supp/ 1:29-38.

32. Halmai Z, Dome P, Vereczkei A, Abdul-Rahman O, Szekely A, Gonda X, et al. Associations between depression severity and purinergic receptor P2RX7 gene polymorphisms. J Affect Disord 2013;150:104-109.

33. Hejjas K, Szekely A, Domotor E, Halmai Z, Balogh G, Schilling B, et al. Association between depression and the Gln460Arg polymorphism of P2RX7 gene: a dimensional approach. Am J Med Genet B Neuropsychiatr Genet 2009; 150B:295-299.

34. Nagy G, Ronai Z, Somogyi A, Sasvari-Szekely $M$, Rahman OA, Mate A, et al. P2RX7 Gln460Arg polymorphism is associated with depression among diabetic patients. Prog Neuropsychopharmacol Biol Psychiatry 2008;32:18841888.

35. Soronen $\mathrm{P}$, Mantere $\mathrm{O}$, Melartin $\mathrm{T}$, Suominen $\mathrm{K}$, Vuorilehto $\mathrm{M}$, Rytsälä $\mathrm{H}$, et al. P2RX7 gene is associated consistently with mood disorders and predicts clinical outcome in three clinical cohorts. Am / Med Genet B Neuropsychiatr Genet 2011;156B:435-447.

36. Basso AM, Bratcher NA, Harris RR, Jarvis MF, Decker MW, Rueter LE. Behavioral profile of $P 2 X 7$ receptor knockout mice in animal models of depression and anxiety: relevance for neuropsychiatric disorders. Behav Brain Res 2009;198:8390.

37. Wilkinson SM, Gunosewoyo H, Barron ML, Boucher A, 
McDonnell M, Turner P, et al. The first CNS-active carborane: a novel $P 2 X 7$ receptor antagonist with antidepressant activity. ACS Chem Neurosci 2014;5:335-339.

38. Jiang LH, Mackenzie AB, North RA, Surprenant A. Brilliant blue G selectively blocks ATP-gated rat P2X(7) receptors. Mol Pharmacol 2000;58:82-88.

39. Ma M, Ren Q, Zhang JC, Hashimoto K. Effects of brilliant blue $G$ on serum tumor necrosis factor-a levels and depression-like behavior in mice after lipopolysaccharide administration. Clin Psychopharmacol Neurosci 2014;12:31-36.

40. Czéh B, Fuchs E, Wiborg O, Simon M. Animal models of major depression and their clinical implications. Prog Neuropsychopharmacol Biol Psychiatry 2016;64:293-310.

41. Willner P. Chronic mild stress (CMS) revisited: consistency and behavioural-neurobiological concordance in the effects of CMS. Neuropsychobiology 2005;52:90-110.

42. Willner P, Muscat R, Papp M. Chronic mild stress-induced anhedonia: a realistic animal model of depression. Neurosci Biobehav Rev 1992;16:525-534.

43. Abelaira HM, Réus GZ, Quevedo J. Animal models as tools to study the pathophysiology of depression. Rev Bras Psiquiatr 2013;35 Suppl 2:S112-S120.

44. Deussing JM. Animal models of depression. Drug Discov Today Dis Models 2006;3:375-383.

45. Wiborg O. Chronic mild stress for modeling anhedonia. Cell Tissue Res 2013;354:155-169.

46. Yang C, Zhou C, Li J, Chen Z, Shi H, Yang W, et al. Quantitative proteomic study of the plasma reveals acute phase response and $L X R / R X R$ and $F X R / R X R$ activation in the chronic unpredictable mild stress mouse model of depression. Mol Med Rep 2018;17:93-102.

47. Liu W, Xue X, Xia J, Liu J, Qi Z. Swimming exercise reverses CUMS-induced changes in depression-like behaviors and hippocampal plasticity-related proteins. I Affect Disord 2018;227:126-135.

48. Csölle C, Baranyi M, Zsilla G, Kittel A, Gölöncsér F, Illes P, et al. Neurochemical changes in the mouse hippocampus underlying the antidepressant effect of genetic deletion of P2X7 receptors. PLoS One 2013;8:e66547.

49. Porsolt RD, Le Pichon M, Jalfre M. Depression: a new animal model sensitive to antidepressant treatments. Nature 1977; 266:730-732.

50. Willner P. Validity, reliability and utility of the chronic mild stress model of depression: a 10-year review and evaluation. Psychopharmacology (Berl) 1997; 134:319-329.

51. Lu M, Yang JZ, Geng F, Ding JH, Hu G. Iptakalim confers an antidepressant effect in a chronic mild stress model of depression through regulating neuro-inflammation and neurogenesis. Int J Neuropsychopharmacol 2014;17:1501-1510.

52. Pereira VS, Casarotto PC, Hiroaki-Sato VA, Sartim AG, Guimarães FS, Joca SR. Antidepressant- and anticompulsivelike effects of purinergic receptor blockade: involvement of nitric oxide. Eur Neuropsychopharmacol 2013;23:1769-1778.
53. Pan $\mathrm{Y}$, Chen XY, Zhang QY, Kong LD. Microglial NLRP3 inflammasome activation mediates IL-1 $\beta$-related inflammation in prefrontal cortex of depressive rats. Brain Behav Immun 2014:41:90-100.

54. Lu K, Wang J, Hu B, Shi X, Zhou J, Tang Y, et al. Brilliant blue $G$ attenuates lipopolysaccharide-mediated microglial activation and inflammation. Neural Regen Res 2013;8:599-608.

55. Wang YC, Cui Y, Cui JZ, Sun LQ, Cui CM, Zhang HA, et al. Neuroprotective effects of brilliant blue Gon the brain following traumatic brain injury in rats. Mol Med Rep 2015;12: 2149-2154.

56. Alcocer-Gómez E, de Miguel M, Casas-Barquero N, Núñez-Vasco J, Sánchez-Alcazar JA, Fernández-Rodríguez A, et al. NLRP3 inflammasome is activated in mononuclear blood cells from patients with major depressive disorder. Brain Behav Immun 2014;36:111-117.

57. Xu Y, Sheng H, Bao Q, Wang Y, Lu J, Ni X. NLRP3 inflammasome activation mediates estrogen deficiency-induced depression- and anxiety-like behavior and hippocampal inflammation in mice. Brain Behav Immun 2016;56: 175-186.

58. Xue J, Li H, Deng X, Ma Z, Fu Q, Ma S. L-Menthone confers antidepressant-like effects in an unpredictable chronic mild stress mouse model via NLRP3 inflammasome-mediated inflammatory cytokines and central neurotransmitters. Pharmacol Biochem Behav 2015;134:42-48.

59. Zhang ZT, Du XM, Ma XJ, Zong Y, Chen JK, Yu CL, et al. Activation of the NLRP3 inflammasome in lipopolysaccharide-induced mouse fatigue and its relevance to chronic fatigue syndrome. J Neuroinflammation 2016;13:71.

60. Li R, Wang X, Qin T, Qu R, Ma S. Apigenin ameliorates chronic mild stress-induced depressive behavior by inhibiting interleukin-1 $\beta$ production and NLRP3 inflammasome activation in the rat brain. Behav Brain Res 2016;296:318-325.

61. Zhang Y, Liu L, Peng YL, Liu YZ, Wu TY, Shen XL, et al. Involvement of inflammasome activation in lipopolysaccharide-induced mice depressive-like behaviors. CNS Neurosci Ther 2014;20:119-124.

62. Sahin C, Albayrak O, Akdeniz TF, Akbulut Z, Yanikkaya Demirel G, Aricioglu F. Agmatine reverses sub-chronic stress induced nod-like receptor protein 3 (NLRP3) activation and cytokine response in rats. Basic Clin Pharmacol Toxicol 2016; 119:367-375.

63. Sahin C, Aricioglu F. Future directions of cytokine hypothesis in depression: 'NLRP3 inflamazomu'. Klin Psikofarmakol Bülteni Bull Clinical Psychopharmacol 2013;23:280-288.

64. Wang Y, Xu J, Liu Y, Li Z, Li X. TLR4-NF- $\kappa B$ signal involved in depressive-like behaviors and cytokine expression of frontal cortex and hippocampus in stressed C57BL/6 and ob/ob mice. Neural Plast 2018;2018:7254016.

65. Peng WF, Fan F, Li X, Zhang QQ, Ding J, Wang X. Different behavioral and pathological changes between epilepsy-associated depression and primary depression models. Epilepsy 
Behav 2018;83:212-218.

66. Wang YL, Han QQ, Gong WQ, Pan DH, Wang LZ, Hu W, et al. Microglial activation mediates chronic mild stress-induced depressive- and anxiety-like behavior in adult rats. J Neuroinflammation 2018;15:21.

67. Kimbler DE, Shields J, Yanasak N, Vender JR, Dhandapani KM. Activation of P $2 X 7$ promotes cerebral edema and neurological injury after traumatic brain injury in mice. PLoS One 2012;7:e41229.

68. Peng W, Cotrina ML, Han X, Yu H, Bekar L, Blum L, et al. Systemic administration of an antagonist of the ATP-sensitive receptor $P 2 X 7$ improves recovery after spinal cord injury. Proc Natl Acad Sci U S A 2009;106:12489-12493.

69. Fehlings MG, Nguyen DH. Immunog/obulin G: a potential treatment to attenuate neuroinflammation following spinal cord injury. J Clin Immunol 2010;30 Suppl 1:S109-S112. 